\title{
IDENTIFYING TARGETS FOR INTERVENTION BY ANALYZING BASINS OF ATTRACTION
}

\author{
MICHAEL P. VERDICCHIO ${ }^{1}$ AND SEUNGCHAN KIM ${ }^{1,2 *}$ \\ ${ }^{1}$ School of Computing, Informatics and Decision Systems Engineering, Ira A. Fulton Schools of Engineering, \\ Arizona State University, Tempe, AZ \\ ${ }^{2}$ Computational Biology Division, Translational Genomics Research Institute (TGen), Phoenix, AZ \\ mv@asu.edu,dolchan@tgen.org
}

\begin{abstract}
Motivation: A grand challenge in the modeling of biological systems is the identification of key variables which can act as targets for intervention. Good intervention targets are the "key players" in a system and have significant influence over other variables; in other words, in the context of diseases such as cancer, targeting these variables with treatments and interventions will provide the greatest effects because of their direct and indirect control over other parts of the system. Boolean networks are among the simplest of models, yet they have been shown to adequately model many of the complex dynamics of biological systems. Often ignored in the Boolean network model, however, are the so called basins of attraction. As the attractor states alone have been shown to correspond to cellular phenotypes, it is logical to ask which variables are most responsible for triggering a path through a basin to a particular attractor.

Results: This work claims that logic minimization (i.e. classical circuit design) of the collections of states in Boolean network basins of attraction reveals key players in the network. Furthermore, we claim that the key players identified by this method are often excellent targets for intervention given a network modeling a biological system, and more importantly, that the key players identified are not apparent from the attractor states alone, from existing Boolean network measures, or from other network measurements. We demonstrate these claims with a well-studied yeast cell cycle network and with a WNT5A network for melanoma, computationally predicted from gene expression data.
\end{abstract}

Keywords: Boolean Networks; Attractors; Logic Minimization; Intervention

\section{Introduction}

Biological systems are complex in many dimensions as endless transportation and communication networks all function simultaneously. While differential equation models are the most comprehensive at capturing and modeling the true dynamic behaviors of a real biological system, ${ }^{1}$ the use of such a framework requires supplying precise model parameters, most of which are not readily measurable with current technologies.

Boolean networks are among the simplest of models, yet they have been shown to adequately model many of the complex dynamics of biological systems. Their popularity is also based on the ease of distilling our knowledge about a particular biological process to positive and negative pair-wise relationships. Since seminal work by Stuart Kauffman in the 1960s relating network attractor states to cell fate, ${ }^{2}$ Boolean network dynamics have been studied and related to various biological phenomena. In addition to Boolean networks, many other graphical models have become popular in the modeling of biological interactions, with one interesting property often being the biological significance of network hubs (though this is also a contested view ${ }^{3}$ ). Specifically, vertices (or nodes) in networks with high degree (also known

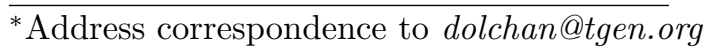


as network hubs) have often been found to have higher biological significance than those less connected nodes in the same network, especially in scale-free networks. Thus, some simple topological analysis, including network centrality measures, can help to identify interesting variables and possibly even targets for intervention.

Wuensche $e^{4}$ and others also have studied basins of attraction in Boolean network models of genomic regulation, specifically the relationship of their structures to the stability of attractors (cell types) in the face of perturbations. However, because of the size and transient nature of basins of attraction, they are often neglected in analysis in favor of the attractor states.

As a basin of attraction is a collection of states leading into a corresponding attractor, i.e. phenotype, careful analysis of these basins could reveal interesting biological characteristics that determine cell fate. In this study we employ a logic reduction algorithm to reduce the Boolean states comprising our basins of attraction to their minimal representations, and it is from these minimizations that we identify intervention targets.

\section{Background}

Despite its simplicity, the Boolean network model has proven to be quite viable at approximating certain aspects of biological processes. For example, it has been used to simulate the yeast cell cycle, ${ }^{5}$ which we look at closely in this work. It has also been used to simulate the expression pattern of segment polarity genes in Drosophila melanogaster ${ }^{6}$ as well as the vocal communication system of the songbird brain. ${ }^{7,8}$ Since we are investigating within a modeling and simulation framework, we employ the often used assumption of synchronous update; however, studies on modeling and analysis of asynchronous update in the context of random Boolean networks can be found. ${ }^{9-12}$

Since Kauffman's seminal work there have been countless variations and extensions of the use of Boolean networks for modeling biological systems, and various inference procedures have been proposed for them. ${ }^{13-15}$ Shmulevich et al. ${ }^{16}$ pioneered work on a stochastic extension to the model called probabilistic Boolean networks (PBNs), which share the rule-based nature of Boolean networks but also handle uncertainty. Within this extended framework of PBNs, studies focusing on external system control were performed by Datta et al. ${ }^{17,18}$ studies by Pal et $a l .{ }^{19}$ and Choudhary et $a l .{ }^{20}$ explored intervention in PBNs to avoid undesirable states.

One major shortcoming of Boolean networks is the exponential growth of the state space with the number of variables, prompting others to work in the Boolean framework itself to achieve some kind of improvement. The approach of Richardson ${ }^{21}$ attempted to shrink the size of the state space through the careful removal of "frozen nodes" and network leaf nodes. The smaller state space then lends itself more readily to the discovery of attractors and basins by sampling methods. Dubrova et $a .^{22}$ explored properties of random Boolean networks, particularly their robustness in the face of topological changes and the removal of "redundant vertices", thus shrinking the state space. While effective in shrinking the space and removing extraneous nodes, neither of these methods is looking for key players in a system or possible intervention targets; in fact both methods have the chance of eliminating such variables.

In an attempt to achieve certain analysis goals, various authors modified or translated the Boolean formalism into another framework. Saez et $a .^{23}$ as well as Schlatter et al. ${ }^{24}$ converted 
their Boolean models of biological systems into hypergraphs, generalizing graphs with edges connecting sets of vertices instead of just pairs or singletons, thus lending themselves to representing Boolean functions. Both papers use analysis techniques to identify important pathways, network motifs and feedback loops. The work of Schlatter et al.also mentions the discovery of relevant hubs in the network. Steggles et al. ${ }^{25}$ employed a classic concept of converting to a different graphical structure, Petri nets. In making this conversion, they used the logic minimization technique we employ (discussed below), albeit in a different way.

Maji and Pradipta ${ }^{26}$ did not use a Boolean network but nonetheless work with the notion of state transition using a related discrete model: fuzzy cellular automata. Their work uses multi-valued logic and presents a new way of identifying attractor basins; however it does not focus on the identification of intervention targets in the system. Mar and Quackenbush ${ }^{27}$ also employed the notion of a state transition space without the direct use of a Boolean network. Using their regression model they strive to classify core variables (genes in their case) as they decompose state space trajectories. Their method, however, is dependent on time-course data, and furthermore its primary focus is at the pathway level and not the variable level.

In this work we stay with the classical formulation of Boolean networks but concentrate on the basins of attraction themselves to identify the key variables in the system. While limited by the exponential complexity inherent to Boolean network state spaces, we work here with tractible network sizes and describe plans to expand to larger networks in the future. Recently, ${ }^{28}$ we successfully used the same yeast network as this study, a human aging network, as well as a version of the WNT5A network for melanoma also presented here in order to study the planning of interventions in biological networks. The intervention targets selected by the Artificial Intelligence planning techniques in that work are in agreement with intervention targets suggested by the methodology presented in this work.

In the coming sections we first formally define our methodology with a sample network and example. Then, we apply our methodology to a well-studied genetic model of the yeast cell cycle. Following this proof of concept we apply our methodology to a WNT5A network computationally predicted from a melanoma gene expression data set. The reader is also referred to our technical report ${ }^{29}$ for an additional application to the aforementioned human aging network. We conclude with some comments on our current and future work.

\section{Methods}

In this section we formally define our methodology. We first briefly summarize the Boolean network formalism and touch upon a basic description of logic reduction. Finally we discuss some measures used in the identification of important variables and intervention targets and then apply all of this to an example network. The reader is referred to our previous technical report $^{29}$ for more on the Boolean network formulation, a smaller example, as well as further description of logic reduction; Xiao and Yufei ${ }^{30}$ also add to the description of Boolean networks.

\subsection{Boolean Networks}

A Boolean network $\mathbf{B}(V, \mathbf{f})$ is made of a set of binary nodes $V=\left\{x_{1}, x_{2}, \cdots, x_{n}\right\}$, where $x_{i} \in 0,1$, and a set of functions $\mathbf{f}=\left\{f_{1}, f_{2}, \cdots, f_{n}\right\}$ that define a state of $\mathbf{x}$ at time $(t+1)$ 
as $x(t+1)=f_{i}\left(x_{i 1}(t), x_{i 2}(t), \ldots, x_{i k_{i}}(t)\right)$, where $f_{i}$ is a Boolean function and $k_{i}$ is called the connectivity of $x_{i}$. The state transition diagram $\mathbf{G}(S, E)$ of a Boolean network $\mathbf{B}(V, \mathbf{f})$ with $n$ nodes is a directed graph where $|\mathrm{S}|=|\mathrm{E}|=2^{n}$. Each of the vertices represents one possible configuration of the $n$ variables in the network and each of the directed edges represents the transition between two states as Boolean functions are synchronously applied to all variables.

In the absence of interventions or perturbations, beginning in any initial state, repeated application of transition functions will bring the network to a finite set of states, $\left\{\mathbf{a}_{1}, \mathbf{a}_{2}, \cdots, \mathbf{a}_{m}\right\} \subseteq S$ and cycle among them forever in fixed sequence. This set of states is known as an attractor, denoted A. An attractor with just one state is called a singleton attractor and an attractor with more than one state is called a cyclic attractor. Boolean networks may have anywhere from one cyclic attractor comprised of $2^{n}$ states to $2^{n}$ point attractors, although most commonly a network will have just a handful of singleton or short-cycle attractors. The complete set of states from which a network will eventually reach $\mathbf{A}$ is known as the basin of attraction for $\mathbf{A}$, denoted $\mathbf{B}=\left\{\mathbf{b}_{1}, \mathbf{b}_{2}, \cdots, \mathbf{b}_{M}\right\} \subseteq S$. All attractors are subsets of their basins (i.e. $\mathbf{A}_{i} \subseteq \mathbf{B}_{i}, \forall_{i}$ ), all basins are mutually exclusive (i.e. $\mathbf{B}_{i} \bigcap \mathbf{B}_{j}=\emptyset, \forall_{i, j}, i \neq j$ ), and the complete state space is comprised entirely of all basins (i.e. $\bigcup_{i} \mathbf{B}_{i}=S$ ). In this study we use the BN/PBN Toolbox ${ }^{31}$ for Boolean network simulation and processing.

\subsection{Logic Minimization}

Logic minimization (or reduction) is a classic problem from digital circuit design employed to reduce the number of actual logic gates needed to implement a given function. ${ }^{32}$ With careful logic minimization one can reduce the number of gates required and thus include more functionality on a single chip. Minimization identifies variables which have no influence on the outcome of a function and marks them appropriately as a don't-care. As a simple example, we take the Boolean function: $(A \wedge B) \vee(\neg A \wedge B)$ (2 signals, 4 gates). Since the role of $A$ changes while $B$ remains $O N$ with the same output, it is clear to see that the only influencing variable is $B$, which can be given with just that signal itself (a single gate).

In this study, we use Espresso, ${ }^{33}$ which is a heuristic logic minimizer designed to efficiently reduce logic complexity even for large problems. We supply as input the set of states in a particular basin of attraction $\left(\mathbf{B}_{i}\right)$; this input comprises the ON-cover (or truth table) in disjunctive normal form (DNF) for a Boolean function whose output is $O N$ for the states of $\mathbf{B}_{i}\left(\left\{\mathbf{b}_{1} \vee \mathbf{b}_{2} \vee \cdots \vee \mathbf{b}_{M_{i}}\right\} \mapsto O N\right)$ and whose output is $O F F$ for the states of $\mathbf{S} \backslash \mathbf{B}_{i}$. Espresso analyzes this cover and returns a minimal (though not necessarily unique) DNF set comprised of one or more terms, denoted $\mathbf{T}_{i}=\left\{\mathbf{t}_{1}, \mathbf{t}_{2}, \cdots, \mathbf{t}_{N_{i}}\right\}$, where $N_{i} \leq M_{i}$. These $\mathbf{t}_{i}$ have some variables set to $O N$, some set to $O F F$, and some set as don't-care. The presence of these don't-care variables in some terms is what allows the reduction.

\subsection{Measures: Popularity, Term Power and Variable Power}

After applying logic minimization to a set of Boolean functions one is left with a minimal DNF representation comprised of a set of terms containing ones, zeros, and don't-cares. We have shown how to spot important variables in a very small example, ${ }^{29}$ but a more formalized method is needed to identify key variables and possible targets for intervention from the 
minimized terms in larger problems. To this end we introduce three simple measures. The first is to measure how frequently a variable $(v)$ is required to be $O N$ or $O F F$ across different terms, called Popularity $(p)$, and is defined as:

$$
p(v)=\frac{z(v)}{N_{i}},
$$

where $z(v)=\sum_{j=1}^{N_{i}} I\left(v, \mathbf{t}_{j}\right), N_{i}$ is the total number of terms in $\mathbf{T}_{i}$, and $I\left(v, \mathbf{t}_{j}\right)$ is an indicator function: 1 when $v$ is ON or OFF in $\mathbf{t}_{j}, 0$ otherwise. Next, we define a measure to identify terms where a few variables demonstrate supremacy over many others. These terms are powerful due to the combinatorial effect of their few set variables. If a five-variable term has one variable set and four listed as don't-cares, that one set variable controls 16 configurations covered by the don't-care variables (half of the state space). This term would be more powerful than a term with two variables set and three don't-cares. Formally, Term Power $\left(P_{T}\right)$ is defined as:

$$
P_{T}(\mathbf{t})=1-\frac{1}{n} \sum_{j=1}^{n} I\left(v_{j}, \mathbf{t}\right),
$$

where $n$ is the number of variables in the term (and network). Term Power is used in calculating our third measure. Given the notion of term power, one can also consider variables which preside over powerful terms to be potentially important and powerful intervention targets. Variable Power $\left(P_{V}\right)$ of a variable $v$ will be defined as the average term power over the terms in which it is explicitly configured, i.e. $v$ is not don't-care:

$$
P_{V}(v)=\frac{1}{z(v)} \sum_{j=1}^{N_{i}} P_{T}\left(\mathbf{t}_{j}\right) \cdot I\left(v, \mathbf{t}_{j}\right)
$$

\subsection{Other Measures to Identify Key Players}

There are various network centrality measures often used in network studies, particularly concerning biological networks, to identify important variables. We have already touched on the degree of a node, but we also consider the network centrality measures of betweenness, centroid value, and eccentricity. High betweenness indicates that a variable is crucial in maintaining connections between other variables. The centroid value for a variable provides a weighted centrality index. A high eccentricity measure indicates that all other nodes are in proximity. Full definitions as well as biological explanations can be found in the supplementary information of Scardoni et al., ${ }^{34}$ but in short, network nodes with high values for these measures can be correlated with biologically significant nodes, possibly even intervention targets.

For Boolean networks, there are also variable-specific measures known as Influence and Sensitivity for a variable $x_{i}$, denoted $r\left(x_{i}\right)$ and $s\left(x_{i}\right)$, respectively. The reader is referred to Shmulevich et al. ${ }^{16,35}$ for formal definitions. In short, in biological Boolean networks, variables with high influence have the potential to regulate the dynamics of the network, and so they are of interest to this study. Sensitivity represents the degree to which a variable is affected by other variables, and so of the most interest are variables with the highest influence and the lowest sensitivity. Since our measures $p$ and $P_{V}$ are specific to each basin, this presents an 
unfair advantage over the network-generality of $r\left(x_{i}\right)$ and $s\left(x_{i}\right)$. Thus, we extend the measures shown in Shmulevich et al. ${ }^{16,35}$ to be specific to a particular basin of attraction by manipulating the joint probability distribution of the state space; we simply assign a zero probability to any state not in the basin and assign a uniform probability to states within.

Example: An 8-variable Boolean network:

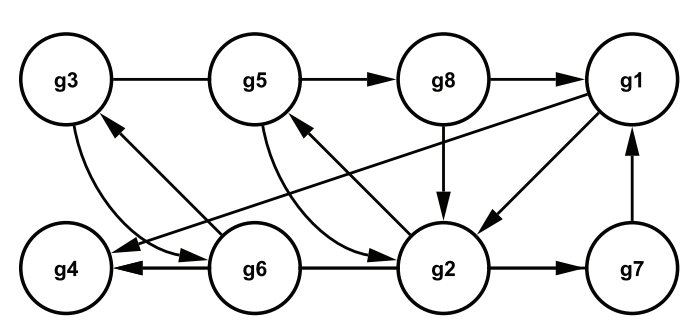

(a) Network

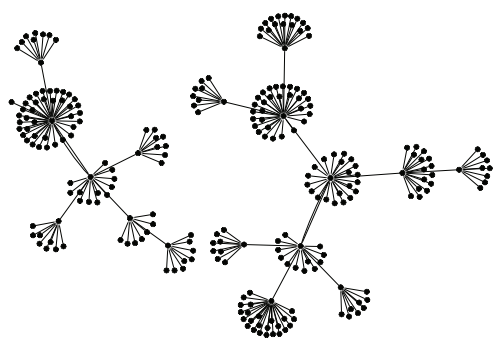

(b) State Transition

Fig. 1: Eight-Variable Example Boolean Network

To show these measures and also our claim regarding the utility of our methodology over other measures, we create the 8-variable network shown in Fig. 1(a), in which we assign at most three random inputs and random Boolean functions. Simulation resulted in two basins of attraction, shown in Fig. 1(b). Basin 1 included 160 states converging on a cyclic attractor of length two ([01011101] and [11011100]), and Basin 2's remaining 96 states converged on another cyclic attractor of length two ([00011100] and [11011101]). Logic reduction reduced the 160 states in Basin 1 to a set of three terms, and the 96 states of Basin 2 to a set of four terms: $\mathbf{T}_{1}=\{[0----00] \vee[1-----10] \vee[1----01]\}, \mathbf{T}_{2}=\{[1-----00] \vee[0-----1-] \vee$ [0-----1] $\vee[-----11]\}$, where "-" indicates a don't-care.

After analysis with the measures defined in the previous section, we find, based on high $p$ and $P_{V}, g 1, g 7$ and $g 8$ to be of interest. Because each of $g 1, g 7$ and $g 8$ are explicitly configured in each of three terms for the larger basin and in 3 out of 4 terms in the smaller basin, their scores for $p$ and $P_{V}$ are each identical and overshadow the remaining variables. In this example, we again observe that simply identifying vertices in the graph with high degree does not necessarily reveal important variables. With self-loops removed to prevent inflation of degree counts, the variables with the highest degree are $g 2$ with six incident edges and $g 1$ with four. From our analysis, $g 1$ is one of the most important variables. However the variable with the highest degree, $g 2$, has been shown to have no influence at all in our analysis. When the network centrality measures of betweenness, centroid value, eccentricity and node degree are calculated for this toy network, we find that $g 8$ is frequently reported with high scores, just like our approach. $r\left(x_{i}\right)$, in fact, identifies $g 1, g 7$ and $g 8$ as important, which match our three best. However, several of the measures, including $s\left(x_{i}\right)$, incorrectly dismiss $g 7$, and many measures also elevate $g 2$, which is shown to have no real intervention capabilities. A table of all measures can be found on the supplementary website; an illustrated expansion of this 
example, along with a simpler one, can be also be found there, and in our previous report. ${ }^{29}$

\section{Results}

In this section we set out to prove the efficacy of our method on real world examples. For this proof of concept we analyze a Boolean network model of the yeast cell cycle and identify significant variables in the system corroborated by its original manuscript. We then demonstrate our approach on a Boolean network not constructed manually, but rather learned from gene expression data directly. In our technical report ${ }^{29}$ we also apply our method to the systems biology of human aging, where we step away from genetic interactions and demonstrate the utility of our method on our Boolean network model for human senescence.

\subsection{Boolean Network Model for Yeast Cell Cycle and Its Analysis}

As a proof of concept on a nonrandom network we will apply our methodology to a wellstudied Boolean network model of the yeast cell cycle ${ }^{5}$ and show that key variables described in the manuscript are identified by our approach. In their paper, Li et al. manually construct a Boolean network modeling the yeast cell cycle using 11 of the most important genes out of the approximately 800 known to play a role in the process. This network is simulated and results in seven basins of attraction, one of which is by far the largest and was studied exclusively in the paper. In this basin of attraction, which included 1,764 states, Li et al. were able to trace the trajectory of the yeast cell cycle from one of the fringe, or "Garden of Eden", ${ }^{4}$ states down to the eventual point attractor state. The Boolean network adapted from Li et al. is shown in on the supplementary website, the original paper, ${ }^{5}$ and our technical report. ${ }^{29}$

After applying logic minimization to these 1,764 states we are left with a sum of 39 product terms. An abstraction of these terms can be seen in Table 1. In the table the terms are seen across columns (sorted by $P_{T}$ ), with ones and zeros represented by black and white, respectively, and don't-cares shown in grey. Some variables are set frequently and others are not. Some terms have many requirements, and others have few. The $p$ and $P_{V}$ measures were calculated for each of the eleven genes in the network. The three most popular variables are Clb5,6, Clb1,2, and Mcm1. The most powerful variable was identified as Cln3.

Table 1: Minimized Yeast Cell Cycle Basin $($ Black $=1$, White $=0$, Grey $=$ don't-care $)$

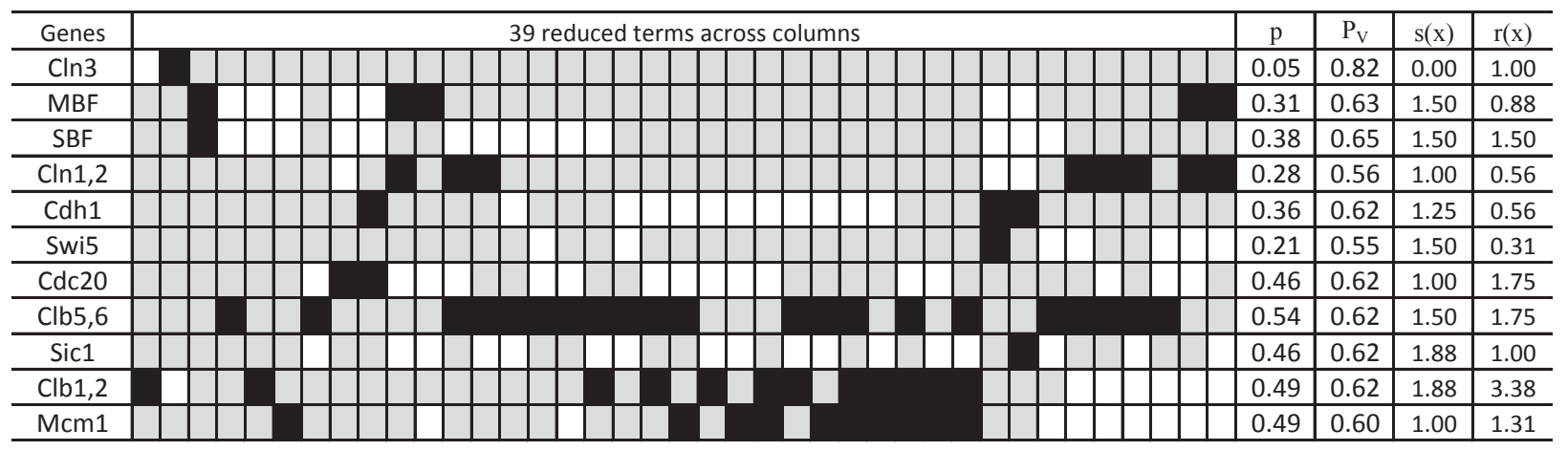


Starting with the most popular variable, we find that Clb5,6 is required to be in a particular state 54 percent of the time. Furthermore we find that in each of the 21 terms in which Clb5, 6 is in a specific configuration, that configuration is $O N$, or active. Since the Clb5 gene (part of the Clb5,6 variable) is described as being responsible for driving the cell into the $\mathrm{S}$ phase (in which the DNA is synthesized and chromosomes are replicated), it seems reasonable to find it strongly represented in the minimized basin. If the role of Clb5 were not known beforehand, analysis of the basin in the manner described could identify it as important (and in the $O N$ state) even though it is $O F F$ in the eventual attractor state.

Next we look at one of the second-most popular variables in the reduced basin, namely Clb1,2. The Clb2 gene (part of the Clb1,2 variable) is stated as being responsible for the transition in and out of the $\mathrm{M}$ phase (in which chromosomes are separated and the cell is divided into two). Thus, like Clb5,6, it is not surprising to find it here among the most frequently specified variables in the basin representing the cell cycle. Unlike Clb5,6, the configuration of Clb1,2 is not consistent - it is found in the OFF configuration 7 times and in the $O N$ configuration 12 times. However, since it is the activation and subsequent degradation of Clb2 which initiates and terminates the $\mathrm{M}$ phase, the split nature of the configurations seems appropriate.

There are other variables with high $p$ which are not explicitly called out in the paper. Given the corroboration of those which are called out in the paper, further investigation of the roles of cyclin inhibitors Cdc20 and Sic1, and of transcription factor Mcm1 is warranted.

Finally we look at the most powerful variable, cyclin Cln3, which was described in the paper as the trigger committing the cell to the division process. Despite its importance, we find it only explicitly configured in 2 of the 39 terms in the reduced basin (once for $O F F$ and once for $O N$ ), which ranks it lowest in the $p$ measure. However, because these two terms are the most powerful, Cln3's $P_{V}$ score is quickly elevated. It is also interesting to find that in these two terms, only one other variable is specifically configured, namely, Clb1,2. In fact, these two variables are in opposite configurations in these two terms; when Cln3 is $O N$, Clb1,2 is $O F F$ and when Cln3 is $O F F, \mathrm{Clb} 1,2$ is $O N$. This is interesting because Cln3 is described as triggering the G1 phase (the starting phase), and Clb1,2 controls the entry and exit from the M phase (the ending phase). Their opposite configurations in the reduced basin terms seem to agree quite harmoniously with their regulatory control at extreme ends of the cell cycle.

When the network centrality measures of betweenness, centroid value, eccentricity and node degree are calculated for this yeast network, we find that Clb1,2 and Clb5,6 are frequently reported with high scores, just like we find using our approach. This is also the case when $r\left(x_{i}\right)$ is calculated based on the Boolean network properties underlying the topology. However, the centrality measures also report variables such as Clb1,2, SBF and MBF, which are shown mathematically by our method to have little intervention power. Furthermore, these measures give little consideration to other key variables, including Cln3 and Mcm1, which our approach mathematically shows to have some intervention capabilities. Thus, our approach reports the key variables described by Li et al. and missed by traditional measures, and avoids reporting mathematically weak variables reported strongly by traditional measures. 


\subsection{Application to WNT5A Network for Melanoma}

After applying our approach to a hand-made network, we applied our methodology to a wellstudied WNT5A network computationally predicted from a melanoma data set. ${ }^{36-38}$ In our previous work, ${ }^{38}$ the original data set was narrowed down to the ten most critical variables; these were selected out of 587 total on the basis of their strong interactive connectivity and either their known or likely roles in WNT5A driven induction of an invasive phenotype in melanoma cells, or their close predictive relationship with these genes. For each of the ten variables, we were able to identify the three most ideal predictors out of the remaining nine. Using this connectivity and a binary quantization of the original data set, the best binary logic functions were inferred for each target minimizing the Bayes error. ${ }^{39,40}$ From these functions, the Boolean network attractors and basins were identified. The reader is referred to the cited publications for detailed information on the data and connectivity, and to the supplementary website for the functions identified, as well as elucidating figures.

Table 2: WNT5A Basin Attractor States (Black =1, White =0) with Basin Measures; $s_{i}(x)$ and $r_{i}(x)$ are basin-specific influence and sensitivity, which are discussed in the next subsection

\begin{tabular}{c|c|c|c|c|c|c|c|c|c|c|c|c|c|c|c|c|c}
\hline & $\mathrm{B} 1$ & $\mathrm{p}$ & $\mathrm{P}_{\mathrm{V}}$ & $\mathrm{s}_{1}(\mathrm{x})$ & $\mathrm{r}_{1}(\mathrm{x})$ & $\mathrm{B} 2$ & $\mathrm{p}$ & $\mathrm{P}_{\mathrm{V}}$ & $\mathrm{s}_{2}(\mathrm{x})$ & $\mathrm{r}_{2}(\mathrm{x})$ & $\mathrm{B} 3$ & $\mathrm{p}$ & $\mathrm{P}_{\mathrm{V}}$ & $\mathrm{s}_{3}(\mathrm{x})$ & $\mathrm{r}_{3}(\mathrm{x})$ & $\mathrm{s}(\mathrm{x})$ & $\mathrm{r}(\mathrm{x})$ \\
\hline WNT5A & 0.45 & 0.57 & 1.79 & 2.25 & & 0.70 & 0.44 & 1.72 & 1.70 & & 1.00 & 0.20 & 1.00 & 2.75 & 1.75 & 2.00 \\
\hline S100B & 0.32 & 0.53 & 1.03 & 0.88 & & 0.40 & 0.40 & 0.96 & 0.59 & 1.00 & 0.20 & 1.25 & 1.25 & 1.00 & 0.75 \\
\hline RET1 & 0.23 & 0.54 & 0.00 & 1.22 & & 0.35 & 0.46 & 0.00 & 1.29 & 0.50 & 0.20 & 0.00 & 1.00 & 0.00 & 1.25 \\
\hline MMP-3 & 0.50 & 0.55 & 0.00 & 0.51 & & 0.60 & 0.48 & 0.00 & 0.47 & & 1.00 & 0.20 & 0.00 & 1.00 & 0.00 & 0.50 \\
\hline Pho-C & 0.27 & 0.53 & 0.96 & 0.24 & & 0.35 & 0.37 & 0.52 & 0.27 & 0.50 & 0.20 & 0.50 & 0.00 & 0.75 & 0.25 \\
\hline MLANA & 0.00 & 0.00 & 1.26 & 0.90 & & 0.00 & 0.00 & 1.24 & 0.58 & 0.00 & 0.00 & 1.00 & 1.00 & 1.25 & 0.75 \\
\hline HADHB & 0.32 & 0.50 & 0.81 & 0.74 & & 0.55 & 0.41 & 0.64 & 0.77 & 1.00 & 0.20 & 3.00 & 0.50 & 0.75 & 0.75 \\
\hline SNCA & 0.68 & 0.54 & 1.65 & 0.30 & & 0.70 & 0.50 & 1.31 & 0.19 & 1.00 & 0.20 & 2.50 & 0.25 & 1.50 & 0.25 \\
\hline STC2 & 0.82 & 0.55 & 1.31 & 1.08 & & 0.75 & 0.47 & 1.19 & 0.92 & 1.00 & 0.20 & 1.00 & 0.25 & 1.25 & 1.00 \\
\hline PIR & 0.86 & 0.55 & 1.79 & 2.48 & & 0.70 & 0.49 & 1.71 & 2.51 & & 1.00 & 0.20 & 1.00 & 3.25 & 1.75 & 2.50 \\
\hline
\end{tabular}

The state space (1,024 states) was partitioned into three basins of attraction: Basin 1 had a singleton attractor state with a total basin size of 544 states, Basin 2 has a two-state cyclic attractor with a total basin size of 472 states, and Basin 3 had a singleton attractor with a total basin size of just 8 states. As seen in Table 2, our measures $p$ and $P_{V}$ reported the intervention capabilities of Pirin, STC2, SNCA, and WNT5A. STC2 is known to interact with MMP-3, ${ }^{41}$ another variable in this network, SNCA is known to be aberrantly hypermethylated in human cancer cells, ${ }^{42}$ it is known that "cytoplasmic localization of PIR may represent a characteristic of WNT5A network for melanoma progression", ${ }^{43}$ and WNT5A has a known role in human melanoma progression. ${ }^{37}$ That three of our top four intervention targets are either melanoma-related or cancer-related speaks well for their true intervention capabilities.

When compared to the network centrality measures, as well as $r\left(x_{i}\right)$ and $s\left(x_{i}\right)$, Pirin and WNT5A were identified by most of them. However, also among the high scoring results for these measures was MLANA, which was shown mathematically by our results to have zero influence on the network dynamics. This is not totally surprising, considering this network is 
derived from melanoma data in which all melanocytes should be present, and that $p$ and $P_{V}$ are basin-specific (see below). While all variables in such a small, carefully selected set will bear some significance, even MLANA, our approach simply reveals those with true intervention capabilities given the topology. Furthermore, some measures dismissed STC2 and SNCA by including it among the lowest scoring variables despite its influence potential.

\subsection{Usefulness of $p$ and $P_{V}$ over Other Measures}

We have seen the ability of $p$ and $P_{V}$ to identify variables with great combinatorial control over the state space of a Boolean network. We have further demonstrated how those variables identified are often known to be suitable targets for intervention. In demonstrating this we have compared $p$ and $P_{V}$ to $r\left(x_{i}\right)$ and $s\left(x_{i}\right)$, as well as network centrality measures, and here we discuss some differences in these measures.

While $r\left(x_{i}\right)$ and $s\left(x_{i}\right)$ are based on Boolean functions, $p$ and $P_{V}$ are based on Boolean states. Influence ${ }^{16}$ is computed by variable pairs in a matrix and summed by rows and columns to get $r\left(x_{i}\right)$ and $s\left(x_{i}\right)$, where $p$ and $P_{V}$ are independent measurements on variables and do not depend on pairs. $r\left(x_{i}\right)$ and $s\left(x_{i}\right)$ are general measures, where $p$ and $P_{V}$ are specific to each basin of attraction. To level the field of comparison, we created a basin-specific version of $r\left(x_{i}\right)$ and $s\left(x_{i}\right)\left(r_{k}\left(x_{i}\right)\right.$ and $s_{k}\left(x_{i}\right)$ for basin $\left.k\right)$, but they were not able to offer any new insight that $r\left(x_{i}\right)$ and $s\left(x_{i}\right)$ were not already able to. To see this, observe the closeness and value and symmetry in dynamics (based on basin size) between the measurements in Table 2 and in the table on the supplementary website for the human aging network.

There are additional advantages over $r\left(x_{i}\right)$ and $s\left(x_{i}\right) . p$ and $P_{V}$ are not only basin-specific, but they are also value-specific. While we can adapt an influence matrix to be basin-specific, it still cannot be made value-specific. Thus, with $p$ and $P_{V}$, because of the minimized terms, we not only know where to intervene, but precisely how to do so. These values, or how we should intervene, can be and often are different than the values in the attractor state (if we're lucky enough to not have a cyclic attractor where values toggle), and furthermore the same target may be viable for more than one basin, but with different values. This kind of information is not available with an influence matrix or the derived measures $r\left(x_{i}\right)$ and $s\left(x_{i}\right)$.

Furthermore, $p$ and $P_{V}$ allow us to find the minimal effective intervention. Any computational aid to intervention studies will always be human-reviewed in the end, so it need not give one definitive answer. We can say with mathematical certainty that setting certain variables together will force a basin (and thus attractor) to be selected. With a set of minimized terms we can find the smallest interventions (highest $P_{T}$ ) using the most effective targets (high $p$ and/or $P_{V}$ ) which are suitable for intervention with current medical abilities (human evaluation of mathematical possibilities).

\section{Conclusion and Future Work}

In this paper, we showed the importance of analyzing Boolean network basins of attraction in identifying targets for intervention. Furthermore, we demonstrated that these targets are not always evident in attractor states themselves, in the network topology, or even from various existing measures, both graph-theoretic and Boolean-network-specific. Our use of logic 
minimization significantly reduces the representation of basins of attraction, and the proposed measures stratify the terms, revealing both the key players and how to manipulate them.

The analysis of the yeast cell cycle network demonstrated that our methodology can identify key variables in the system. We were able to systematically identify three important variables described specifically by the original study and propose others for further study. Our application to the WNT5A network for melanoma demonstrated the applicability of our approach beyond hand-created networks to networks inferred from biological data; furthermore our targets identified for intervention had been previously validated by laboratory studies.

This approach is most appropriate to smaller hand-made or high-confidence networks due to the size complexity issues in Boolean networks. Current efforts involve overcoming the scalability issues inherent in enumerating complete state spaces, which quickly becomes intractable. We are investigating approximation approaches to identify attractor states and enumerate most of their basins. We intend to take full advantage of high performance computing clusters, both in terms of memory and parallelization. We also are working on expanding our implementations and measures to handle multi-valued logic, taking us beyond the Boolean constraint and allowing even more levels of abstraction.

\section{Supplementary Material}

http://biocomputing.asu.edu/basinreduction/psb2011/

\section{Acknowledgement}

This study funded partly by NIH R21LM009706 (MV, SK) and SFAZ CAA 0243-08 (SK).

\section{References}

1. J. Goutsias and S. Kim, Biophys J 86, 1922 (April 2004).

2. S. Kauffman, Journal of Theoretical Biology 22, 437 (March 1969).

3. G. Lima-Mendez and J. Helden, Mol. BioSyst. 5, 1482 (December 2009).

4. A. Wuensche, Genomic regulation modeled as a network with basins of attraction., in Pacific Symposium on Biocomputing, 1998.

5. F. Li, T. Long, Y. Lu, Q. Ouyang and C. Tang, Proceedings of the National Academy of Sciences of the United States of America 101, 4781 (April 2004).

6. R. Albert and H. G. Othmer, Journal of Theoretical Biology 223, 1 (July 2003).

7. J. Yu, V. A. Smith, P. P. Wang, A. J. Hartemink and E. D. Jarvis, Bioinformatics 20, bth448 (July 2004).

8. V. A. Smith, E. D. Jarvis and A. J. Hartemink, Bioinformatics 18, S216 (July 2002).

9. C. Gershenson, Classification of random boolean networks, in ICAL 2003: Proceedings of the eighth international conference on Artificial life, (MIT Press, Cambridge, MA, USA, 2003).

10. K. Klemm and S. Bornholdt, Physical Review E 72, 055101+ (Nov 2005).

11. F. Greil and B. Drossel, Physical Review Letters 95, 048701+ (Jul 2005).

12. X. Deng, H. Geng and M. Matache, Biosystems 88, 16 (March 2007).

13. T. Akutsu, S. Miyano and S. Kuhara, Pacific Symposium on Biocomputing, 17 (1999).

14. I. Shmulevich, A. Saarinen, O. Yli-Harja and J. Astola, Inference of genetic regulatory networks via best-fit extensions, in Computational and Statistical Approaches to Genomics, eds. W. Zhang and I. Shmulevich (Kluwer Academic Publishers, Boston, 2003) pp. 197-210. 
15. H. Lähdesmäki, I. Shmulevich and O. Yli-Harja, Machine Learning 52, 147 (July 2003).

16. I. Shmulevich, E. R. Dougherty, S. Kim and W. Zhang, Bioinformatics 18, 261 (February 2002).

17. A. Datta, A. Choudhary, M. L. Bittner and E. R. Dougherty, Machine Learning 52, 169 (July 2003).

18. A. Datta, A. Choudhary, M. L. Bittner and E. R. Dougherty, Bioinformatics 20, 924 (April 2004).

19. R. Pal, A. Datta, M. L. Bittner and E. R. Dougherty, Bioinformatics 21, 1211 (April 2005).

20. A. Choudhary, A. Datta, M. L. Bittner and E. R. Dougherty, Bioinformatics 22, 226 (January 2006).

21. K. A. Richardson, Advances in Complex Systems 8, 365 (2005).

22. E. Dubrova, M. Teslenko and H. Tenhunen, A computational scheme based on random boolean networks, in Transactions on Computational Systems Biology X, eds. C. Priami, F. Dressler, O. B. Akan and A. Ngom, 2008) pp. 41-58.

23. J. Saez-Rodriguez, L. G. Alexopoulos, J. Epperlein, R. Samaga, D. A. Lauffenburger, S. Klamt and P. K. Sorger, Molecular Systems Biology 5 (December 2009).

24. R. Schlatter, K. Schmich, I. Avalos Vizcarra, P. Scheurich, T. Sauter, C. Borner, M. Ederer, I. Merfort and O. Sawodny, PLoS Comput Biol 5, e1000595+ (December 2009).

25. L. J. Steggles, R. Banks, O. Shaw and A. Wipat, Bioinformatics 23, 336 (February 2007).

26. P. Maji, Fundam. Inf. 86, 143 (2008).

27. J. C. Mar and J. Quackenbush, PLoS Comput Biol 5, e1000626+ (December 2009).

28. D. Bryce, M. P. Verdicchio and S. Kim, ACM Transactions on Intelligent Systems and Technology (To Appear).

29. M. P. Verdicchio and S. Kim, Reduction of Boolean Network Basins of Attraction Reveals Intervention Targets, tech. rep., Arizona State University (Tempe, AZ, 2010).

30. Y. Xiao, Current Genomics 10, 511 (November 2009).

31. I. Shmulevich, E. R. Dougherty and W. Zhang, Bioinformatics 18, 1319 (October 2002).

32. A. Marcovitz, Introduction to Logic Design, first edn. (McGraw-Hill, Feb 2002).

33. R. L. Rudell and A. L. Sangiovanni-Vincentelli, Espresso-mv: Algorithms for multiple valued logic minimization, in Proc. of the IEEE Custom Integrated Circuits Conference, 1985.

34. G. Scardoni, M. Petterlini and C. Laudanna, Bioinformatics 25, 2857 (November 2009).

35. I. Shmulevich and S. A. Kauffman, Physical Review Letters 93, 048701+ (Jul 2004).

36. M. Bittner, P. Meltzer, Y. Chen, Y. Jiang, E. Seftor, M. Hendrix, M. Radmacher, R. Simon, Z. Yakhini, A. Ben-Dor, N. Sampas, E. Dougherty, E. Wang, F. Marincola, C. Gooden, J. Lueders, A. Glatfelter, P. Pollock, J. Carpten, E. Gillanders, D. Leja, K. Dietrich, C. Beaudry, M. Berens, D. Alberts and V. Sondak, Nature 406, 536 (August 2000).

37. A. T. Weeraratna, Y. Jiang, G. Hostetter, K. Rosenblatt, P. Duray, M. Bittner and J. M. Trent, Cancer Cell 1, 279 (April 2002).

38. S. Kim, H. Li, E. R. Dougherty, N. Cao, Y. Chen, M. Bittner and E. B. Suh, Journal of Biological Systems 10, 337 (2002).

39. E. R. Dougherty, S. Kim and Y. Chen, Signal Processing 80, 2219 (2000).

40. S. Kim, E. R. Dougherty, M. L. Bittner, Y. Chen, K. Sivakumar, P. Meltzer and J. M. Trent, Journal of biomedical optics 5, 411 (October 2000).

41. J. Y. Y. Sung, S. M. M. Park, C.-H. H. Lee, J. W. W. Um, H. J. J. Lee, J. Kim, Y. J. Oh, S.-T. T. Lee, S. R. Paik and K. C. C. Chung, The Journal of biological chemistry 280, 25216 (July 2005).

42. A. Y. Law, K. P. Lai, C. K. Ip, A. S. Wong, G. F. Wagner and C. K. Wong, Experimental cell research 314, 1823 (May 2008).

43. S. Licciulli, C. Luise, A. Zanardi, L. Giorgetti, G. Viale, L. Lanfrancone, R. Carbone and M. Alcalay, BMC cell biology $\mathbf{1 1}, 5+$ (January 2010). 